\title{
A Pink Conjunctival Lesion
}

\author{
Uma Lesão Conjuntival Rosada
}

\section{Helena P. FILIPE $\triangle^{1}$, Cristina VAZ-PEREIRA ${ }^{1}$, Silvestre CRUZ $^{1}$ \\ Acta Med Port 2015 Nov-Dec;28(6):784-784}

Keywords: Conjunctival neoplasms; Granuloma, pyogenic.

Palavras-chave: Granuloma piogénico; Neoplasias da conjuntiva.

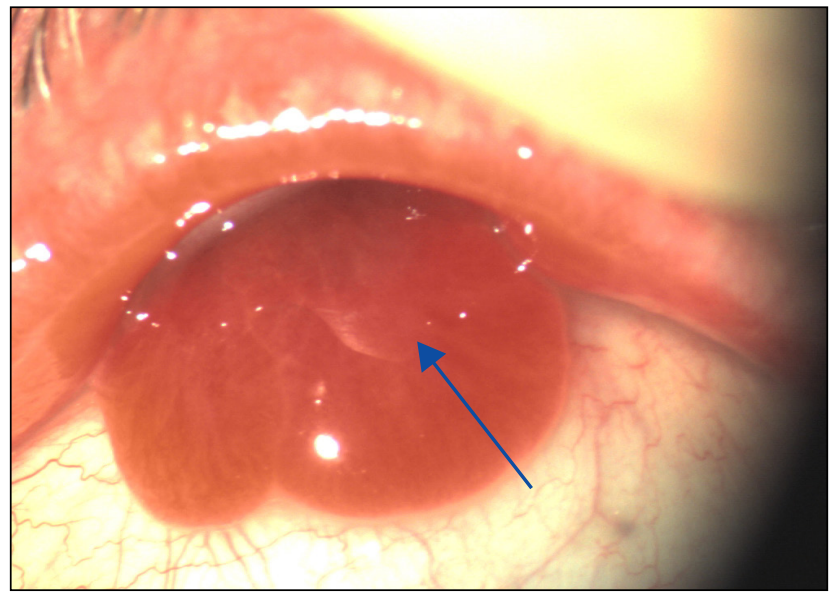

Figure 1 - Smooth, pedunculated, salmon-pink, bulbar conjunctival lesion

A 65-year old, farm worker female with a six weeks right eye (RE) discomfort, tearing and progressive eyelid swelling was referred to our clinic. Past ophthalmic and systemic history was uneventful.

She presented painless, considerable RE superior eyelid edema, bilateral best-corrected visual acuity of 0.9 , normal ocular movement and pupil reflex. Slit lamp examination revealed conjunctival hyperemia, upper perilimbal keratitis and a painless, smooth, pedunculated, salmon-pink, bulbar conjunctival lesion (Fig.1). Ophthalmoscopy and ultrasound examination were unremarkable. At $3 \mathrm{~mm}$ distance, a long

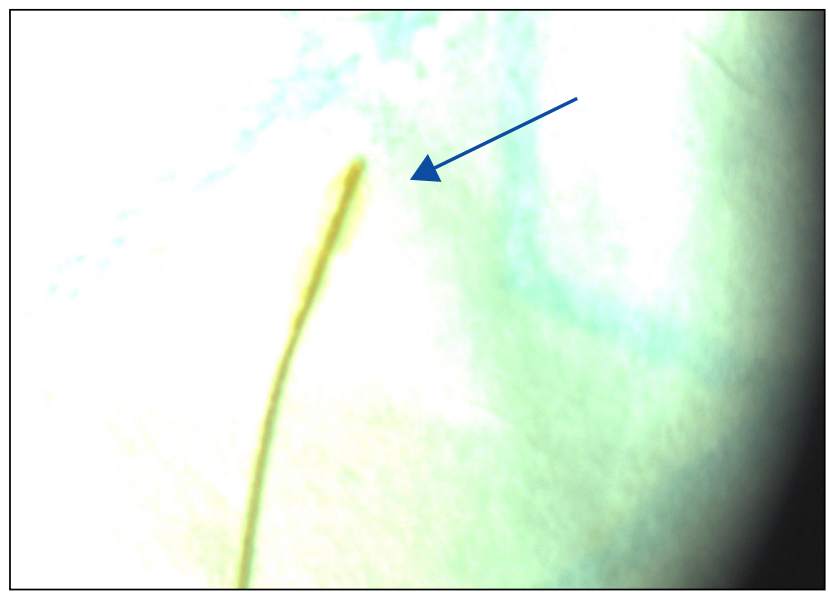

Figure 2 - Hay straw

'suture thread like' was pulled out with a forceps under topical anesthesia. It proved to be a hay straw (Fig.2).

Topical treatment (ofloxacin, dexamethasone and carmellose) was started. Two weeks later signs and symptoms have completely subsided.

Despite possible macroscopic similarities with other conjunctival tumors, conjunctival lymphoma included, and absence of eye trauma or surgery past story, the clinical context suggested conjunctival pyogenic granuloma / lobular capillary hemangioma related to a foreign body..$^{1-3}$

\section{REFERENCES}

1. Shields JA, Mashayekhi A, Kligman BE, Kunz WB, Criss J, Eagle RC Jr, et al. Vascular tumors of the conjunctiva in 140 cases. Ophthalmology. 2011;118:1747-53.

2. da Cunha LP, Barros JN, Motono M, Costa FD, da Cunha MC, Chojniak MM. Conjunctival follicular lymphoma after treatment for invasive squamous cell carcinoma. Case Rep Ophthalmol. 2014;5:444-50.

3. Olivier JF. Common conjunctival lesions. Conjunctival lesions present commonly in primary care. CME. 2013:31

\footnotetext{
1. Departamento de Oftalmologia. Instituto Dr. Gama Pinto. Lisboa. Portugal.

$\square$ Autor correspondente: Helena P. Filipe. hpriorfilipe@gmail.com

Recebido: 07 de Abril de 2015 - Aceite: 21 de Julho de 2015 | Copyright @ Ordem dos Médicos 2015
} 


\section{A Pink Conjunctival Lesion}

Acta Med Port 2015:28:784-784

Publicado pela Acta Médica Portuguesa, a Revista Científica da Ordem dos Médicos

Av. Almirante Gago Coutinho, 151

1749-084 Lisboa, Portugal.

Tel: +351218428 215

E-mail: submissao@actamedicaportuguesa.com

www.actamedicaportuguesa.com

ISSN:0870-399X | e-ISSN: 1646-0758

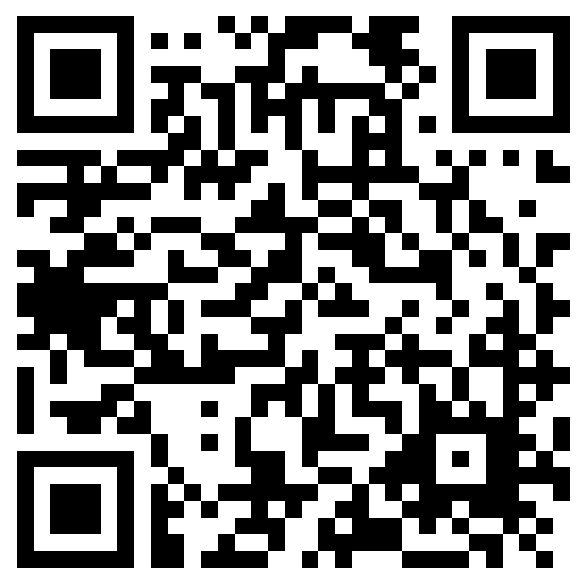

